\title{
X-Ray Reflectivity Study of the Surface of Liquid Gallium
}

\section{Citation}

Kawamoto, E. H., S. Lee, Peter S. Pershan, M. Deutsch, N. Maskil, and B. M. Ocko. 1993. X-ray reflectivity study of the surface of liquid gallium. Physical Review B 47(11): 6847-6850.

\section{Published Version}

doi:10.1103/PhysRevB.47.6847

\section{Permanent link}

http://nrs.harvard.edu/urn-3:HUL.InstRepos:10357542

\section{Terms of Use}

This article was downloaded from Harvard University's DASH repository, and is made available under the terms and conditions applicable to Other Posted Material, as set forth at http:// nrs.harvard.edu/urn-3:HUL.InstRepos:dash.current.terms-of-use\#LAA

\section{Share Your Story}

The Harvard community has made this article openly available.

Please share how this access benefits you. Submit a story.

\section{Accessibility}




\title{
X-ray reflectivity study of the surface of liquid gallium
}

\author{
E. H. Kawamoto, S. Lee, ${ }^{*}$ and P. S. Pershan \\ Division of Applied Science and Department of Physics, Harvard University, Cambridge, Massachusetts 02138 \\ M. Deutsch and N. Maskil \\ Department of Physics, Bar-Ilan University, Ramat-Gan 52100, Israel \\ B. M. Ocko \\ Department of Physics, Brookhaven National Laboratory, Upton, New York 11973
}

(Received 8 January 1993)

\begin{abstract}
$\mathrm{X}$-ray reflectivity from the surface of liquid gallium was measured under ultrahigh vacuum conditions using a novel technique for curved surfaces. The small deviations between the measured and theoretical Fresnel reflectivity for an ideally sharp flat interface for wave-vector transfer $\lesssim 0.5 \AA^{-1}$ imply an interfacial width for the electron density profile of $\lesssim 1.3 \pm 0.2 \AA$. This is consistent with a model of atomic close packing which lacks structure along the surface normal at length scales $>10 \AA$.
\end{abstract}

The properties and structure of the free surface of liquid metals depend sensitively on the many-body nature of the interactions and intra-actions between the ions and the electrons, including subtle electron screening effects. Over the last two decades, research on liquid metals has intensified because of their immense practical and basic scientific importance. Nevertheless, our current understanding of their surfaces is still relatively primitive. ${ }^{1-4}$ Despite a wealth of data on the interfacial surface tension $\gamma$, neither its magnitude nor its variation from material to material has received a satisfactory theoretical explanation. The surface energy of liquid metals can be $\sim 20 \%$ less than that of the solid at the melting temperature $T_{m}$; just above $T_{m}$, the temperature derivative of the surface energy ("surface entropy") is significantly lower than that of the solid. Extensive simulations, ${ }^{5}$ and recent perturbation $^{6}$ and asymptotic Euler-Lagrange ${ }^{7}$ calculations also predict the formation of positionally ordered layers of atoms at the surface. Yet, except for a few isolated experimental results the surface order of liquid metals has not been extensively studied.

Both Rice and others have performed x-ray reflectivity measurements on liquid-metal surfaces. ${ }^{5}$ Unfortunately, without the advantages of synchrotron x-ray sources these studies were inconclusive. They implied interfacial widths that were significantly broader than expected from microscopic considerations, as well as structure along the surface normal at unphysical lengths. In contrast, recent studies of the surfaces of a number of nonmetallic liquids obtained interfacial widths and characteristic lengths in good agreement with simple physical expectations. ${ }^{8,9}$ The experiment we report here demonstrates that the interfacial width between liquid gallium and its vapor is not so anomalously broad as to preclude x-ray reflectivity measurements at the large wave-vector transfers needed to probe surface-induced atomic layering. ${ }^{10}$ In the present measurements there was no evidence for structure at length scales exceeding $10 \AA$.

The geometry of $x$-ray specular reflectivity has been discussed elsewhere. ${ }^{11}$ When the wave-vector transfer $Q$ along the surface normal exceeds about $5 Q_{c}$ $\left[Q_{c}=(4 \pi / \lambda) \sin \theta_{c}\right.$, where $\theta_{c}$ is the critical angle for total external reflection of $x$ rays of wavelength $\lambda]$, the ratio between the measured x-ray reflectivity $R(Q)$ from a rough surface and the theoretical Fresnel reflectivity $R_{F}(Q)$ from an ideal interface is given by

$$
R(Q) / R_{F}(Q) \approx\left|\left(1 / \rho_{\infty}\right) \int d z[\partial\langle\rho(z)\rangle / \partial z] e^{i Q z}\right|^{2}
$$

Here, $\rho_{\infty}$ is the bulk electron density and $\langle\rho(z)\rangle$ represents the dependence of the horizontal planeaveraged electron density on the surface normal coordinate $z .^{8}$ For $Q \lesssim 5 Q_{c}$, an equivalent numerical result can be obtained. ${ }^{12}$ Common to nearly all measurements on disordered surfaces of nonmetallic liquids is that, for $Q^{-1}$ much larger than atomic or molecular dimensions, $R(Q) / R_{F}(Q) \approx \exp \left(-Q^{2} \sigma^{2}\right)$. This implies $\partial\langle\rho(z)\rangle /$ $\partial z \propto \rho_{\infty} \exp \left(-z^{2} / 2 \sigma^{2}\right)$, where $\sigma$ is a measure of the width of the liquid-vapor interface. ${ }^{8}$ Surface-induced atomic layering of period $L$ can cause $R / R_{F}$ to deviate from the Gaussian law (with maximum deviation when $Q L \sim \pi$ ); for liquid metals $L$ is predicted to be of the order of the atomic diameter $D_{a}$. For $Q \ll\left(2 \pi / D_{a}\right) \sim 2 \AA^{-1} \mathrm{x}$ rays merely probe $\langle\rho(z)\rangle$ averaged over $\Delta z \sim 1 / Q$ and since our range of wave-vector transfer was restricted to $Q \leq 0.55 \AA^{-1}$ the data are relatively insensitive to surface-induced atomic layering.

For free surfaces of simple liquids, which have been shown to possess a $\langle\rho(z)\rangle$ whose derivative is Gaussian, $\sigma$ is well represented by some combination of an intrinsic width $\sigma_{\text {int }}$ as well as a thermal fluctuation term $\sigma_{\text {th }}$. The intrinsic width is given by the bulk correlation length $\xi$ which is typically of the order of the atomic (or molecular) radius, while $\sigma_{\text {th }} \sim\left(k_{B} T / \gamma\right)^{1 / 2}$ in the classical limit, where $\gamma$ is the macroscopic surface tension. ${ }^{8}$ For $\mathrm{H}_{2} \mathrm{O}$, $\gamma \approx 73$ dyn $\mathrm{cm}^{-1}$, implying that the fluctuation term is the dominant contribution to the measured value of $\sigma \approx 2.8 \AA$. Although one possible measure of the length scale for the transition from the bulk to vapor density can 
be obtained from simple energy considerations (i.e., $\sim \gamma \kappa_{T}$ where $\kappa_{T}$ is the bulk isothermal compressibility) this estimate is typically three or four times smaller than $\xi{ }^{2}$ Since $\sigma_{\text {int }}$ are of the order $1 \AA$, the net interfacial width for $\mathrm{H}_{2} \mathrm{O}$ and other nonmetallic liquids should be dominated by the fluctuation term. For nearly all the nonalkali liquid metals the values of $\gamma$ are almost an order of magnitude larger than that of water, suggesting that $\sigma_{\text {th }}$ should also be about $1 \AA$; however, since these combine in quadrature the measured interfacial width $\sigma_{\text {meas }} \approx \sqrt{\sigma_{\text {int }}^{2}+\sigma_{\text {th }}^{2}}$ should not be much larger than $\sigma_{\text {int }}$. Nevertheless, the only reported experimental values are considerably larger than expected from experience with simple liquids [i.e., 2.1 $\AA$ (2X) for liquid $\mathrm{Hg}$ and $3.9 \AA$ $(4 \times)$ for liquid $\mathrm{Ga}$. If this discrepancy were typical $R(Q)$ would be unobservably small in the range of $Q$ where interference maxima from surface-induced atomic layering are expected. In contrast, the value of $\sigma=1.3 \pm 0.2 \AA$ we report here is consistent with measurable reflectivities at $Q \approx 2.6 \AA^{-1}$, near the first peak in the $\mathrm{Ga}$ liquid structure factor. ${ }^{13}$

$\mathrm{X}$-ray reflectivity measurements of liquid-metal surfaces pose some technical problems. First, the sample must remain horizontal while being moved vertically to intercept the incident beam that is deflected downward by $\alpha$. Since some of measurements must be made at relatively small angles $\left(\theta_{c} \approx 0.35^{\circ}\right)$, very small errors in sample height can move the beam unacceptably far from the center of the horizontal sample surface. Second, for a beam of height $h \approx 0.04 \mathrm{~mm}$ incident at $0.37^{\circ}$ relative to the horizontal, the angle of incidence relative to the surface will vary by $\pm 1.2^{\circ}$ if the liquid surface has a $150-\mathrm{mm}$ radius of curvature. The combined effects of large surface tension (a problem for small samples) and mechanically excited surface waves (a problem for deep samples) frustrate efforts to attain this degree of flatness for liquid-metal sample surfaces. Bosio et al. ${ }^{14}$ attempted to extract reflectivity data from the curved surface of a liquid-Hg drop, but were unsuccessful. The present work employed a variation of their method in combination with the more traditional specular reflection geometry.

The problem of mechanically excited surface waves was overcome by using shallow enough samples (i.e., $\lesssim 0.3 \mathrm{~mm}$ ) that surface waves with wavelengths longer than the sample depth were suppressed by viscous drag at the liquid/solid interface. ${ }^{8}$ Initially prepared under high vacuum, the substrates, $30-\mathrm{mm}$-diam stainless-steel flats, were sputtered free of oxides in an rf glow discharge sustained in an argon atmosphere, through which liquid gallium (99.9999\% bulk purity) was later dropped onto the substrate surface. The Ga spontaneously spread to cover the sputtered surface with contact angles $\sim 10^{\circ}$, as judged by eye. These samples were transported through air into a UHV chamber where $x$-ray measurements were made at $\mathrm{O}_{2}$ partial pressures less than $10^{-11}$ Torr. The visible oxide film that formed on the surface during transfer was removed with a mechanical scraper, after which residual oxides and other trace impurities were removed by sputtering with 2-keV argon ions. Although surface cleanliness could not be probed in situ, the efficacy of this procedure in producing an oxide-free surface was tested

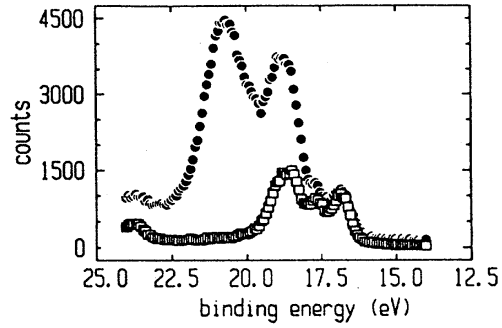

FIG. 1. X-ray photoelectron spectra of the surface of a liquid-Ga-In eutectic alloy atop a stainless-steel substrate. The filled circles are the sum of ten scans from 24- to $14-\mathrm{eV}$ binding energy, showing $3 d$ peaks of elemental $\mathrm{Ga}$ at $19.0 \mathrm{eV}$ as well as those of $\mathrm{Ga}_{2} \mathrm{O}_{3}$ at $20.7 \mathrm{eV}$. Following $20 \mathrm{~s}$ of $15-\mathrm{keV} \mathrm{Ar}^{+}$bombardment, four scans over the same energy window (open squares) show no trace of the oxide peak.

by studying a similarly prepared sample, also transferred through air, in a dedicated $\mathrm{x}$-ray photoelectron spectrometer (XPS). Figure 1 shows the region of the Ga $3 d$ peak in the XPS from the surface of a Ga-In eutectic alloy (24 wt \% In) before and after 20-s bombardment with $15-\mathrm{keV}$ $\mathrm{Ar}^{+}$; the absence of the $\mathrm{Ga}_{2} \mathrm{O}_{3} 3 d$ peak indicates $\lesssim 2-4 \%$ of a monolayer of oxide.

$\mathrm{X}$-ray reflectivity measurements were made on the Harvard-Brookhaven liquid-surface spectrometer installed on beam line X-22B, details of which are described elsewhere. ${ }^{11}$ The incident beam of wavelength $\lambda=1.612$ $\AA$ was collimated to a vertical angular divergence to 0.05 mrad using a $0.5-\mathrm{mm}$ slit immediately preceding the toroidal mirror. Using a $0.03-\mathrm{mm}$-high defining slit 200 mm upstream of the sample, the beam height at the sample position was $0.04 \mathrm{~mm}$. Its width was $0.4 \mathrm{~mm}$ with a $0.1-\mathrm{mrad}$ horizontal angular divergence. As shown schematically in Fig. 2, the incident beam is deflected downward by an angle $\alpha$, striking the top of a rounded drop. Specular reflection of $x$ rays from different positions along the drop yields outgoing rays at upward angles $\beta=\alpha-2 \phi$, where $\phi$ is the angle in the plane of incidence between the vertical and the surface normal at the point where the beam intercepts the top of the drop. Intensity is measured as a function of $\beta$ by vertically scanning a detector with a 2-mm-high slit located 600 $\mathrm{mm}$ from the sample. Confirmation that the signal was due to specular reflection came from horizontal scans of a 1-mm-wide detector slit through both direct and reflected

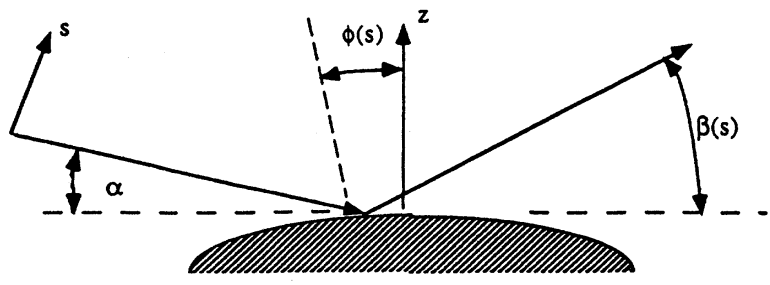

FIG. 2. Schematic diagram of curved liquid-surface kinematics. The parallel incident beam at angle $\alpha$ to the horizontal with an intensity distribution $I_{0}(s)$ strikes the surface at a point where the local normal is at an angle $\phi(s)$ to the vertical. $\mathrm{X}$ rays reflected from this point are detected at an angle $\beta(s)$. 
beams for each $\alpha$, as well as from vertical scans of the sample position at fixed $\alpha$ and $\beta$ with open detector slits.

The vertical intensity distribution of the incident beam $I_{0}(s) \Delta s$ is well represented by a square wave with negligible angular divergence: $I_{0}(s)=1$ for $|s| \lesssim 0.02 \mathrm{~mm}$ and zero otherwise. Hence, the intensity $I(\beta) \Delta \beta$ detected at outgoing angle $\beta$ ( $\Delta \beta \approx \frac{2}{600}$ is the detector's angular resolution) can be interpreted quantitatively. Intensity observed at $\beta$ arises from a point on the sample for which the surface normal is tilted in the plane of incidence from the vertical by $\phi=(\alpha-\beta) / 2$, with resultant scattering angle $2 \theta=(\alpha+\beta)$. Then $I(\beta) \Delta \beta=I_{0}(s) R(Q)|d s / d \beta| \Delta \beta$, where $Q \approx(2 \pi / \lambda)(\alpha+\beta)$. Neglecting the angular dispersion of the incident beam, the liquid surface with curvature radius $R_{0}$ maps the position $s$ along the incident wave front (and with it, the tilt angle $\phi$ at the point where the beam strikes the sample) onto $\beta$ via $|d s / d \beta| \approx\left(R_{0} / 4\right)(\alpha+\beta)$, so that $I(\beta) \Delta \beta \propto Q R(Q)$.

Figure $3(\mathrm{a})$ shows $I(\beta) \Delta \beta$ plotted as a function of $Q$ for $\alpha \approx 0.37^{\circ}\left(\theta_{c} \approx 0.35^{\circ}\right)$. The solid line illustrates the best fit of $Q R_{F}(Q)$ to the data in the range $0.025 \lesssim Q \lesssim 0.075$ $\AA^{-1}$, corresponding to $\Delta \phi \approx 6.4 \mathrm{mrad}$; the only adjustable parameters in this fit were the absolute intensity and a small offset that allowed for the $\pm 0.03^{\circ}$ uncertainty in $\alpha$. Deviations from $Q R_{F}(Q)$ outside this range are probably due to the $0.04-\mathrm{mm}$ height of the beam since, for $\alpha \approx 0.37^{\circ}$, the projection of $I_{0}(s)$ on the incident beam on the horizontal is $\approx(0.04 \mathrm{~mm}) / \sin \left(0.37^{\circ}\right)$ or $6.5 \mathrm{~mm}$, which would span $\sim 41 \mathrm{mrad}$, or $\sim 2.3^{\circ}$, if $R_{0}$ were only $150 \mathrm{~mm}$; from data taken at larger $\alpha$, to be discussed below, $R_{0} \gtrsim 320 \mathrm{~mm}$. The filled squares in Fig. 3(b) are excerpts from the same data, divided by $Q$ and normalized to unity at $Q_{c}$, while the thin solid line represents $R_{F}(Q)$. Since the relative values of data and $R_{F}(Q)$ agree over more than two orders of magnitude, we can conclude that $\exp \left[-(0.1 \sigma)^{2}\right] \gtrsim 0.9$, or $\sigma \lesssim 3.2 \AA$; however, with increasing $\alpha$ the range of $\Delta \phi$ illuminated by the beam decreases and we show below that it is possible to measure absolute reflectivities which place an upper bound on $\sigma$ at $1.3 \pm 0.2 \AA$.

The open circles identified as (c), (d), (e), and (f) in Fig. 3 represent $\beta$ scans for $\alpha \approx 1.8^{\circ}, 2.6^{\circ}, 3.3^{\circ}$, and $4.1^{\circ}$ $\left(Q=0.25,0.35,0.45\right.$, and $\left.0.55 \AA^{-1}\right)$. The open squares above each of these scans represent the backgroundsubtracted integrated intensity for each, while the heavy solid lines represent the convolution of $R_{F}(Q)$ with resolution functions appropriate to the widths of the associated $\beta$ scans. Although the curvature-dependent variation in the orientation of the surface normal is responsible for the widths of these scans their integrated intensities do not depend on $R_{0}$. Aside from the last point at $Q=0.55$ $\AA^{-1}$, all of these measurements would be consistent with $R(Q)=R_{F}(Q)$ and $\sigma=0$. The broken line in Fig. 2 represents a convolution of $R(Q)$ with the distribution in $\phi$ as determined from the data in Figs. 3(c)-3(f), with $R_{F}(Q) \exp \left(-\sigma^{2} Q^{2}\right)$ using the best fit value of $\sigma=1.3 \AA$. The quoted value of $\Delta \sigma= \pm 0.2 \AA$ is obtained by varying $\sigma$ such that the model will go through either point (e) or (f). Although theoretical models of liquid-metal clusters predict surface-induced oscillations in $\langle\rho(z)\rangle$ that are

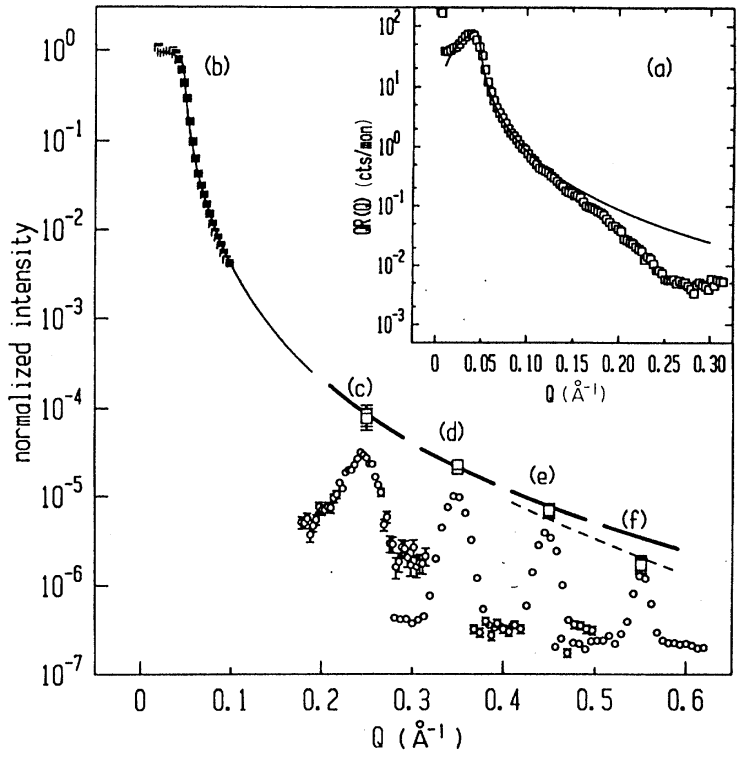

FIG. 3. (a) Plot of $I(\beta) \Delta \beta$ for $\alpha=0.37^{\circ}$. The abscissas are converted to the corresponding $Q$ value and represented by open squares. The solid line shows a fit of $Q R_{F}(Q)$ between 0.025 and $0.075 \AA^{-1}\left(|\phi| \leq 0.18^{\circ}\right)$. (b) Excerpt of data from (a) divided by $Q$ and normalized to unity (filled squares). (c) $-(\mathrm{f})$ Open circles are detector height scans at $0.25,0.35,0.45$, and $0.55 \AA^{-1}$; open squares are background-subtracted integrated intensities of those peaks. Heavy solid line segments denote $R_{F}(Q)$ calculated using Gaussian resolution functions with half widths at half maximum of $0.016,0.010,0.009$, and $0.007 \AA^{-1}$, respectively, corresponding to widths of the scans; broken line segments show $R_{F}(Q) \exp \left(-\sigma^{2} Q^{2}\right)$ with $\sigma=1.3 \AA$.

more or less symmetric about $\rho_{\infty}$, they average out over a length scale of $\sim 10 \AA$, which corresponds to the smallest length scale to which our data are sensitive. ${ }^{5}$ It follows that unless $\langle\rho(z)\rangle$ takes on values substantially greater than $\rho_{\infty}$ near the surface, this value of $\sigma$ is independent of the existence of surface order.

In summary, x-ray reflectivity was measured from the surface of liquid $\mathrm{Ga}$ for wave-vector transfers $Q \lesssim 0.55$ $\AA^{-1}$. The data would not be consistent with an electron density profile along the surface normal having a Gaussian width $\sigma$ in excess of $1.3 \pm 0.2 \AA^{-1}$. Specular x-ray reflectivity would have to be extended to $Q \sim 2.5 \AA^{-1}$ in order to determine the existence of surface-induced atomic layering; based on these results so far, such studies seem practical. This bound on $\sigma$ is compatible with the theoretically expected contribution of $\sim 0.83 \AA$ (at $Q_{z} \approx 0.45 \AA^{-1}$ with in-plane resolution $\Delta Q_{x} \Delta Q_{y}$ $\approx 0.032 \times 0.0004 \AA^{-1}$ ) from thermal excitations if the intrinsic width is $\lesssim \sqrt{\left(1.3^{2}-0.83^{2}\right)}=1.0 \AA$. It is not clear what value to anticipate for the intrinsic width; however, since this value is larger than typical estimates based on the product $\gamma \kappa_{T}$ and smaller than the $1.5-\AA$ atomic radius, we believe that the surface is shown to be atomically flat.

The paucity of data on liquid-metal interfaces can largely be attributed to difficulties in obtaining sufficiently large, flat surfaces and suppressing mechanically excited 
surface waves. In this work we have shown that quantitative measurements can be made on curved samples. Hopefully, the results and techniques presented here will lead to more experiments on the surfaces of both pure metals and alloys in the liquid state.

This work has been supported by grants from the U.S. Department of Energy, No. DE-FG02-88-ER45379 and the National Science Foundation, Nos. DMR-89-20490 and DMR-91-13782. M.D. and N.M. acknowledge the support of the U.S.-Israel Binational Science Foundation, Jerusalem, Israel. S.L. acknowledges support from the Pohang Light Source, Pohang, Korea. The National Synchrotron Light Source at Brookhaven National Laboratory is supported by DOE Contract No. DE-ACO2$76 \mathrm{CH} 00016$.
*Present address: Department of Physics, Ajou University, 5 Wonchun-dong, Kwonson-gu, Suwon 441-749, South Korea.

${ }^{1}$ M. Shimoji, Liquid Metals: An Introduction to the Physics and Chemistry of Metals in the Liquid State (Academic, London, 1977).

${ }^{2}$ N. H. March, Liquid Metals, Concepts and Theory (Cambridge University Press, Cambridge, 1990).

${ }^{3}$ T. E. Faber, Introduction to the Theory of Liquid Metals (The University Press, Cambridge, 1972).

${ }^{4}$ R. Evans, J. Phys. (Paris) 41, C8 (1980).

${ }^{5}$ See the following review and the references contained therein: S. A. Rice, Proc. Natl. Acad. Sci. U.S.A. 84, 4709 (1987).

${ }^{6}$ M. A. Gómez and E. Chacón, Phys. Rev. B 46, 723 (1992).

${ }^{7}$ M. Iwamatsu and S. K. Lai, J. Phys. Condens. Matter 4, 6039 (1992).

${ }^{8}$ See the following review and the references contained therein: P. S. Pershan, Faraday Discuss. Chem. Soc. 89, 231 (1990).

${ }^{9}$ M. K. Sanyal, S. K. Sinha, K. G. Huang, and B. M. Ocko, Phys. Rev. Lett. 66, 628 (1991).
${ }^{10}$ Gallium was chosen for this study because its vapor pressure is sufficiently low that UHV techniquues can be used to maintain an atomically clean surface. For example, below approximately $600^{\circ} \mathrm{C}$ the vapor pressure for liquid gallium is less than $10^{-8}$ Torr. In addition, its low melting temperature, $29.7^{\circ} \mathrm{C}$, simplified the design of the experiment. Furthermore, the temperature range for which gallium is liquid is largest of all known materials, making it one of the more interesting liquid metals.

${ }^{11}$ D. K. Schwartz, M. L. Schlossman, and P. S. Pershan, J. Chem. Phys. 96, 2356 (1992).

${ }^{12}$ L. G. Parratt, Phys. Rev. 95, 359 (1954).

${ }^{13}$ Power-law decay of the intrinsic density profile towards the bulk value can only be detected through more precise lowangle measurements than are possible with the present curved samples.

${ }^{14}$ L. Bosio, R. Cortès, M. Denozière, and G. Folcher, J. Phys. (Paris) 50, C7 (1989). 